\title{
Generalized bending of a plate with a circular inclusion of arbitrary rigidity
}

\section{W Bert}

School of Aerospace and Mechanical Engineering, The University of Oklahoma, Norman, Oklahoma 73019-1052, USA

\begin{abstract}
The paper presents an exact closed-form solution for a thin isotropic plate containing a circular elastic inclusion and subjected to arbitrary bending moments at large distances from the hole. Quantitative results are presented for the bending stress concentration factors for the plate and for the inclusion.
\end{abstract}

Keywords: inclusion, plate bending, stress concentration

\section{NOTATION}

$a \quad$ radius of the inclusion (see Fig. 1)

$a_{1}, b_{1}, A_{i}, B_{i}, C_{i}, F_{i}$

constants of integration

$B \quad$ biaxial bending-moment ratio $\equiv M_{y} / M_{x}$

$D_{i} \quad$ flexural rigidity $=E_{i} h^{3} /\left[12\left(1-v_{i}^{2}\right)\right]$

$E_{i} \quad$ modulus of elasticity

$k \quad=D_{2} / D_{1}$

$K_{i} \quad$ bending stress concentration factor

$\left(M_{r}\right)_{i}, \quad$ bending stress couples in $r, \theta$ coordinates

$\left(M_{\theta}\right)_{i}$

$\left(M_{r} \theta\right)_{i} \quad$ twisting stress couple in $r, \theta$ coordinates

$M_{x}, M_{y} \quad$ bending stress couples in $x, y$ coordinates

$\left(Q_{r}\right)_{i} \quad$ radial-edge transverse shear stress resultant

$r \quad$ radial position coordinate (see Fig. 1)

$\left(V_{r}\right)_{i} \quad$ effective radial-edge shear stress resultant

$w_{i} \quad$ normal deflection

$x, y \quad$ rectangular Cartesian coordinates (see Fig. 1)

$\theta \quad$ angular position coordinate (see Fig. 1)

$v_{i} \quad$ Poisson's ratio

$\rho \quad=r / a$

$\nabla^{2} \quad$ Laplacian operator

\section{Subscripts}

$i=1 \quad$ plate

$i=2 \quad$ inclusion

\section{INTRODUCTION}

The problem of a large thin isotropic plate containing a

The MS was received on 7 March 2000 and was accepted after revision for publication on 22 September 2000. stress-free circular hole and subjected to either uniaxial bending moment or cylindrical bending was solved by Bickley [1] in 1924. Recently, this work was generalized by the present author [2] to the case of generalized bending. The problem of a large plate containing a rigid or elastic inclusion and subjected to three bending loading states and transverse shear was solved by Goland [3] in 1943.

The objective of the present work is to generalize the work in references [2] and [3] to the case of a large plate with an isotropic elastic inclusion subjected to a generalized bending moment field.

\section{ANALYSIS}

The geometry of the problem is a thin isotropic plate containing a circular inclusion at the origin. Cartesian $(x$, $y)$ and polar $(r, \theta)$ coordinates are measured in the midplane of the plate from the centre-line of the inclusion (Fig. 1). A far-field bending moment state having principal bending moments $M_{x}$ and $M_{y}$ per unit length is considered. The biaxial bending-moment ratio is defined as

$$
B \equiv \frac{M_{y}}{M_{x}}
$$

The values of $B$ for various special cases are listed in Table 1 , in which $v_{1}$ denotes Poisson's ratio of the plate.

Let subscripts 1 and 2 refer to the plate and the inclusion respectively. Then, the general expression for the dimensionless deflection of the plate can be expressed in plane polar coordinates $(r, \theta)$ as 


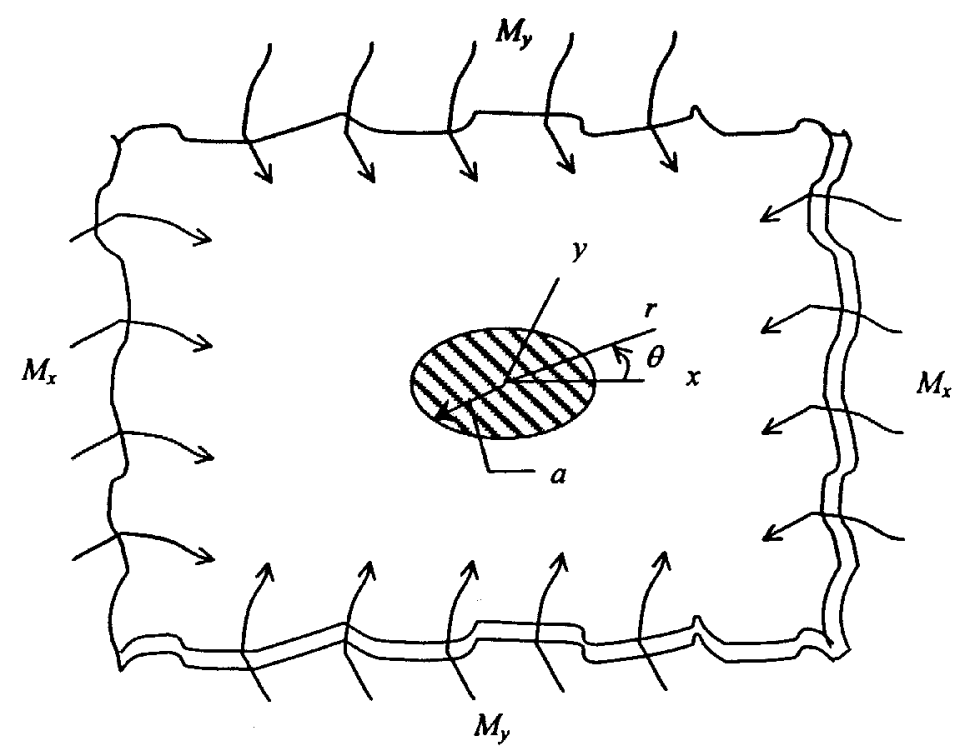

Fig. 1 A thin plate containing a circular inclusion and subjected to bending stress couples $M_{x}$ and $M_{y}$ at great distances from the centre of the inclusion

Table 1 Values of biaxial bendingmoment ratio for various loading cases

\begin{tabular}{ll}
\hline Loading case & $B$ \\
\hline Uniaxial bending stress & 0 \\
Cylindrical bending (uniaxial & $v_{1}$ \\
$\quad$ bending strain) & 1 \\
$\begin{array}{l}\text { Balanced biaxial bending } \\
\quad \text { axisymmetric) }\end{array}$ & \\
Pure twisting & -1 \\
\hline
\end{tabular}

$$
\begin{aligned}
\frac{w_{1}}{a}=-\frac{M_{x} a}{2 D_{1}}[ & a_{1} \rho^{2}+b_{1} \rho^{2} \cos (2 \theta)+A_{1} \ln \rho \\
& \left.+B_{1} \cos (2 \theta)+C_{1} \rho^{-2} \cos (2 \theta)+F_{1}\right]
\end{aligned}
$$

where $a$ is the radius of the inclusion, $a_{1}, b_{1}, A_{1}, B_{1}, C_{1}$ and $F_{1}$ are constants to be determined, $D_{1}$ is the plate flexural rigidity and $\rho \equiv r / a$.

For the inclusion, which is assumed to be solid, i.e. not to contain a hole, the dimensionless deflection is

$$
\frac{w_{2}}{a}=-\frac{M_{x} a}{2 D_{2}}\left[A_{2} \rho^{2}+B_{2} \rho^{2} \cos (2 \theta)+C_{2} \rho^{4} \cos (2 \theta)\right]
$$

where $A_{2}, B_{2}$ and $C_{2}$ are constants to be determined and $D_{2}$ is the inclusion flexural rigidity.

The boundary conditions at great distances from the inclusion ( $\rho$ increasing without bound) are that $M_{x}$ must be finite, that the transverse shear force $Q_{x}$ must vanish at $\theta=0 \mathrm{rad}$, that $M_{y}=B M_{x}$ must be finite and that $Q_{y}$ must vanish at $\theta=\pi / 2$. Thus,

$$
\begin{aligned}
& M_{r}=\frac{M_{x}}{2}[(1+B)+(1-B) \cos (2 \theta)] \\
& M_{r} \theta=-\frac{M_{x}}{2}(1-B) \sin (2 \theta), \quad Q_{r}=0
\end{aligned}
$$

To relate the plate displacement $w_{1}$ to the bending and twisting moments, the constitutive relations

$$
\begin{aligned}
& M_{r}=-\frac{D_{1}}{a^{2}}\left(w_{, \rho \rho}+v_{1} \rho^{-1} w_{, \rho}+v_{1} \rho^{-2} w_{, \theta \theta}\right) \\
& M_{r \theta}=-\left(1-v_{1}\right) \frac{D_{1}}{a^{2}}\left(\frac{w}{\rho}\right)_{, \rho \theta}
\end{aligned}
$$

are used. Combining equations (2), (4), and (5), it is found that

$$
a_{1}=\frac{1+B}{2\left(1+v_{1}\right)}, \quad b_{1}=\frac{1-B}{2\left(1-v_{1}\right)}
$$

The interfacial continuity conditions which must be satisfied at the interface $(\rho=1)$ are matching deflection, radial slope, radial bending moment and radial effective shear force:

$$
\begin{aligned}
& w_{1}(1, \theta)=w_{2}(1, \theta) \\
& \frac{\partial w_{1}}{\partial r}(1, \theta)=\frac{\partial w_{2}}{\partial r}(1, \theta)
\end{aligned}
$$




$$
\begin{aligned}
& \left(M_{r}\right)_{1}(1, \theta)=\left(M_{r}\right)_{2}(1, \theta) \\
& \left(V_{r}\right)_{1}(1, \theta)=\left(V_{r}\right)_{2}(1, \theta)
\end{aligned}
$$

Satisfaction of equation (7) leads to the following two relations:

$$
\begin{aligned}
& A_{2}=\frac{D_{2}}{D_{1}}\left(a_{1}+F_{1}\right) \\
& B_{2}+C_{2}=\frac{D_{2}}{D_{1}}\left(b_{1}+B_{1}+C_{1}\right)
\end{aligned}
$$

Solution of equation (8) yields the two relations

$$
\begin{aligned}
& A_{2}=\frac{D_{2}}{D_{1}}\left(a_{1}+\frac{A_{1}}{2}\right) \\
& B_{2}+2 C_{2}=\frac{D_{2}}{D_{1}}\left(b_{1}-C_{1}\right)
\end{aligned}
$$

Since the radial bending stress couple is

$$
\left(M_{r}\right)_{i}=-D_{i}\left[\frac{\partial^{2} w_{i}}{\partial r^{2}}+v_{i}\left(\frac{1}{r} \frac{\partial w_{i}}{\partial r}+\frac{1}{r^{2}} \frac{\partial^{2} w_{i}}{\partial \theta^{2}}\right)\right]
$$

where $i=1,2$, satisfaction of equation (9) gives the following two equations:

$$
\begin{aligned}
& 2\left(1+v_{2}\right) A_{2}=2\left(1+v_{1}\right) a_{1}-\left(1-v_{1}\right) A_{1} \\
& \left(1-v_{2}\right) B_{2}+6 C_{2}=\left(1-v_{1}\right) b_{1}-2 v_{1} B_{1}+3\left(1-v_{1}\right) C_{1}
\end{aligned}
$$

The effective radial-edge shear force is

$$
\left(V_{r}\right)_{i}=\left(Q_{r}\right)_{i}+\frac{1}{r} \frac{\partial\left(M_{r} \theta\right)_{i}}{\partial \theta}
$$

where

$$
\begin{aligned}
& \left(Q_{r}\right)_{i}=-D_{i} \frac{\partial}{\partial r}\left(\nabla^{2} w_{i}\right) \\
& \left(M_{r} \theta\right)_{i}=-\left(1-v_{i}\right) D_{i}\left(\frac{1}{r} \frac{\partial^{2} w_{i}}{\partial r \partial \theta}-\frac{1}{r^{2}} \frac{\partial w_{i}}{\partial \theta}\right) \\
& \nabla^{2}=\frac{\partial^{2}}{\partial r^{2}}+\frac{1}{r} \frac{\partial}{\partial r}+\frac{1}{r^{2}} \frac{\partial^{2}}{\partial \theta^{2}}
\end{aligned}
$$

Thus, satisfaction of equation (10) requires that

$$
\begin{aligned}
2\left(1-v_{2}\right) B_{2}-6\left(1+v_{2}\right) C_{2}= & 2\left(1-v_{1}\right) b_{1}-2\left(3-v_{1}\right) B_{1} \\
& -6\left(1-v_{1}\right) C_{1}
\end{aligned}
$$

Equations (11) to (17) constitute seven equations in the seven unknown coefficients. Their solutions are as follows:

$$
\begin{aligned}
& A_{1}=\frac{1+B}{1+v_{1}} \frac{1+v_{1}-\left(1+v_{2}\right) k}{1-v_{1}+\left(1+v_{2}\right) k} \\
& A_{2}=\frac{1+B}{1+v_{1}} \frac{1}{1-v_{1}+\left(1+v_{2}\right) k} \\
& B_{1}=\frac{1-B}{1-v_{1}} \frac{1-v_{1}-\left(1-v_{2}\right) k}{3+v_{1}+\left(1-v_{2}\right) k} \\
& B_{2}=\frac{1-B}{1-v_{1}} \frac{2}{3+v_{1}+\left(1-v_{2}\right) k} \\
& C_{1}=-\frac{B_{1}}{2}, \quad C_{2}=0, \quad F_{1}=\frac{A_{1}}{2}
\end{aligned}
$$

where

$$
k \equiv \frac{D_{2}}{D_{1}}
$$

Since

$$
\left(M_{\theta}\right)_{i}=-D_{i}\left(\frac{1}{r} \frac{\partial w_{i}}{\partial r}+\frac{1}{r^{2}} \frac{\partial^{2} w_{i}}{\partial \theta^{2}}+v_{i} \frac{\partial^{2} w_{i}}{\partial r^{2}}\right)
$$

it can be shown that

$$
\begin{aligned}
\frac{\left(M_{\theta}\right)_{1}}{M_{x}}= & \left(1+v_{1}\right) a_{1}+\frac{1}{2}\left(1-v_{1}\right) A_{1} \rho^{-2}+\left[\left(-1+v_{1}\right) b_{1}\right. \\
& \left.-2 B_{1} \rho^{-2}-3\left(1-v_{1}\right) C_{1} \rho^{-4}\right] \cos (2 \theta)
\end{aligned}
$$

and

$$
\frac{\left(M_{\theta}\right)_{2}}{M_{x}}=\left(1+v_{2}\right) A_{2}+\left[-\left(1-v_{2}\right) B_{2}+6 v_{2} C_{2} \rho^{2}\right] \cos (2 \theta)
$$

The bending moment stress concentration factors for the plate $(i=1)$ and inclusion $(i=2)$ are defined as

$$
K_{i}=\frac{\left(M_{\max }\right)_{i}}{M_{x}}
$$

where $\left(M_{\max }\right)_{i}$ is the maximum value of $\left(M_{r}\right)_{i},\left(M_{\theta}\right)_{i}$ and $\left(M_{r} \theta\right)_{i}$.

For biaxial ratios in the range $-1 \leqslant B \leqslant 1$, the maximum values of moment are the circumferential values at the interfacial radius $(\rho=1)$ at an orientation $\theta=\pi / 2$. Thus, 


$$
K_{i}=\frac{\left(M_{\theta}\right)_{i}(1, \pi / 2)}{M_{x}}
$$

Finally,

$$
K_{1}=1+\frac{1}{2}\left(1-v_{1}\right) A_{1}+2 B_{1}+3\left(1-v_{1}\right) C_{1}
$$

and

$$
K_{2}=\left(1+v_{2}\right) A_{2}+\left(1-v_{2}\right) B_{2}
$$

\section{NUMERICAL RESULTS AND DISCUSSION}

Numerical values of $K_{1}$ and $K_{2}$ are given in Figs 2 and 3 respectively for various values of the dimensionless material parameters $v_{1}, v_{2}$ and $k$ and bending moment ratio $B$.

It can be seen that both the $K_{1}$ and the $K_{2}$ curves have a value of unity when the stiffness ratio $D_{2} / D_{1}=1$. This makes sense because then the entire plane is homogeneous, i.e. there is no discontinuity.

When the stiffness ratio $D_{2} / D_{1}=0$, the inclusion vanishes and the plate contains simply a hole. Then the plate stress concentration $K_{1}$ depends upon the biaxial ratio $B$ and the equation coincides exactly with that given in reference [2], which reduces to those given by Bickley [1] for the cases that he considered. The inclusion stress concentration factor vanishes for $D_{2} / D_{1}$, because in fact there is no inclusion present in this case.

As the inclusion stiffness is increased, the stress concentration $K_{1}$ in the plate decreases (Fig. 2) while that $\left(K_{2}\right)$ in the inclusion increases. It is interesting to note that the various families of curves for $K_{2}$ cross at $D_{2} / D_{1}=1$. Thus, the effects of the biaxial ratio $B$ reverse at this point. For instance, for $D_{2} / D_{1}=0.5$, the largest value of $K_{2}$ corresponds to $B=1$ while, for $D_{2} / D_{1}=2$, the largest value of $K_{2}$ corresponds to $B=-1$. In contrast, the various curves for $K_{1}$ converge at $D_{2} / D_{1}=1$ but they do not cross, i.e. they do not reverse.

As a practical example, suppose that a plate has a hole. Now determine the effects of inserting an inclusion and of changing the biaxial ratio. It can be seen from Fig. 2 that the stiffer the inclusion (the higher the $D_{2} / D_{1}$ ratio), the lower is the bending stress ratio $K_{1}$; for instance, for $B=0$ and $D_{2} / D_{1}=3, K_{1}$ is only 0.5 . This means that the presence of a stiff inclusion reduces the maximum bending moment from approximately to $1.7 M_{x}$ to $0.5 M_{x}$. The most beneficial biaxial ratio $B$ for a $D_{2} / D_{1}$ ratio of 3 is $B=-1$, which reduces the maximum bending moment to 0.3 approximately.

This question may arise: what is the effect of purely Poisson ratio mismatch, i.e. $D_{2} / D_{1}=1$ but $v_{2} \neq v_{1}$ ? As a

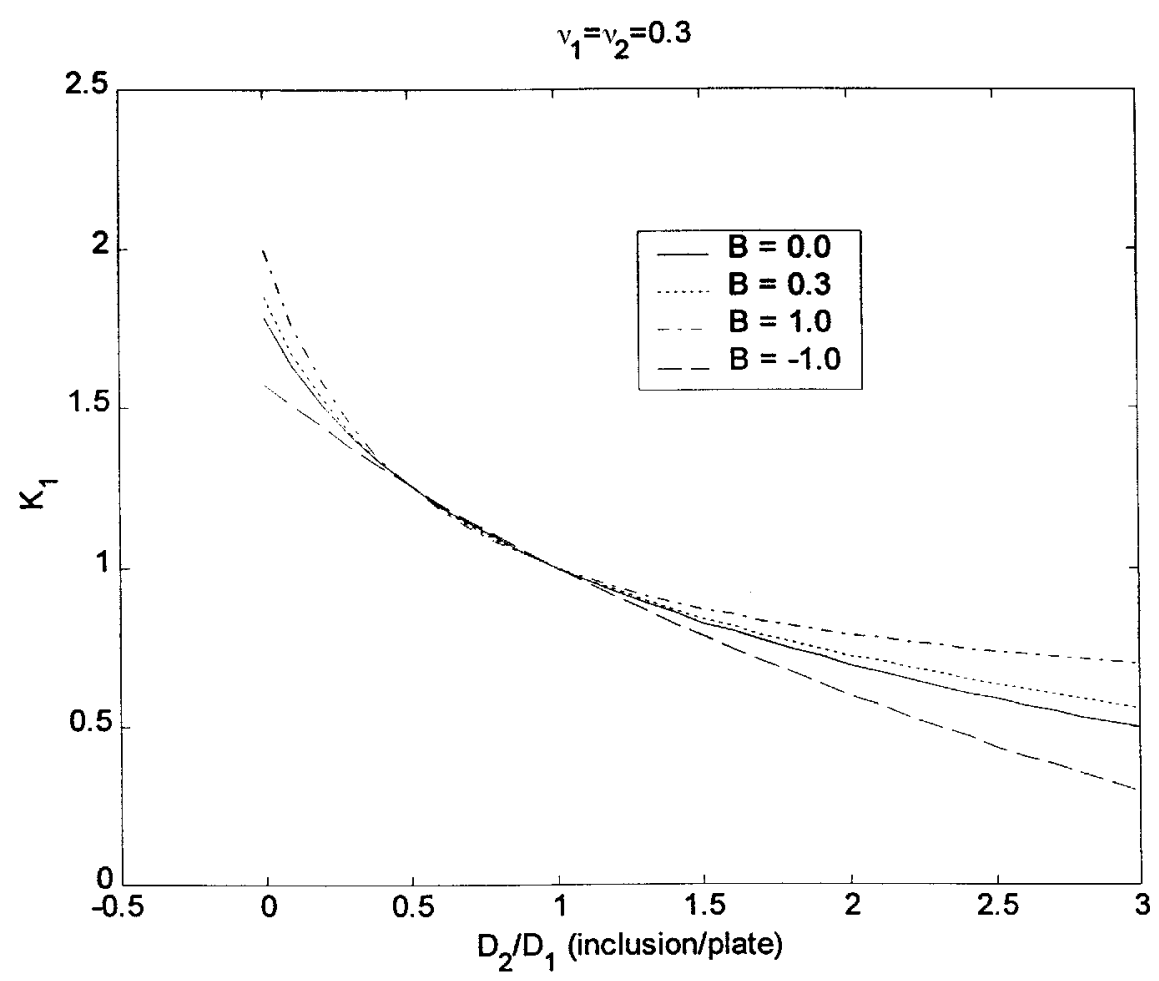

Fig. 2 Dimensionless maximum circumferential bending moment in the plate as a function of the ratio of the flexural rigidity of the inclusion to that of the plate 


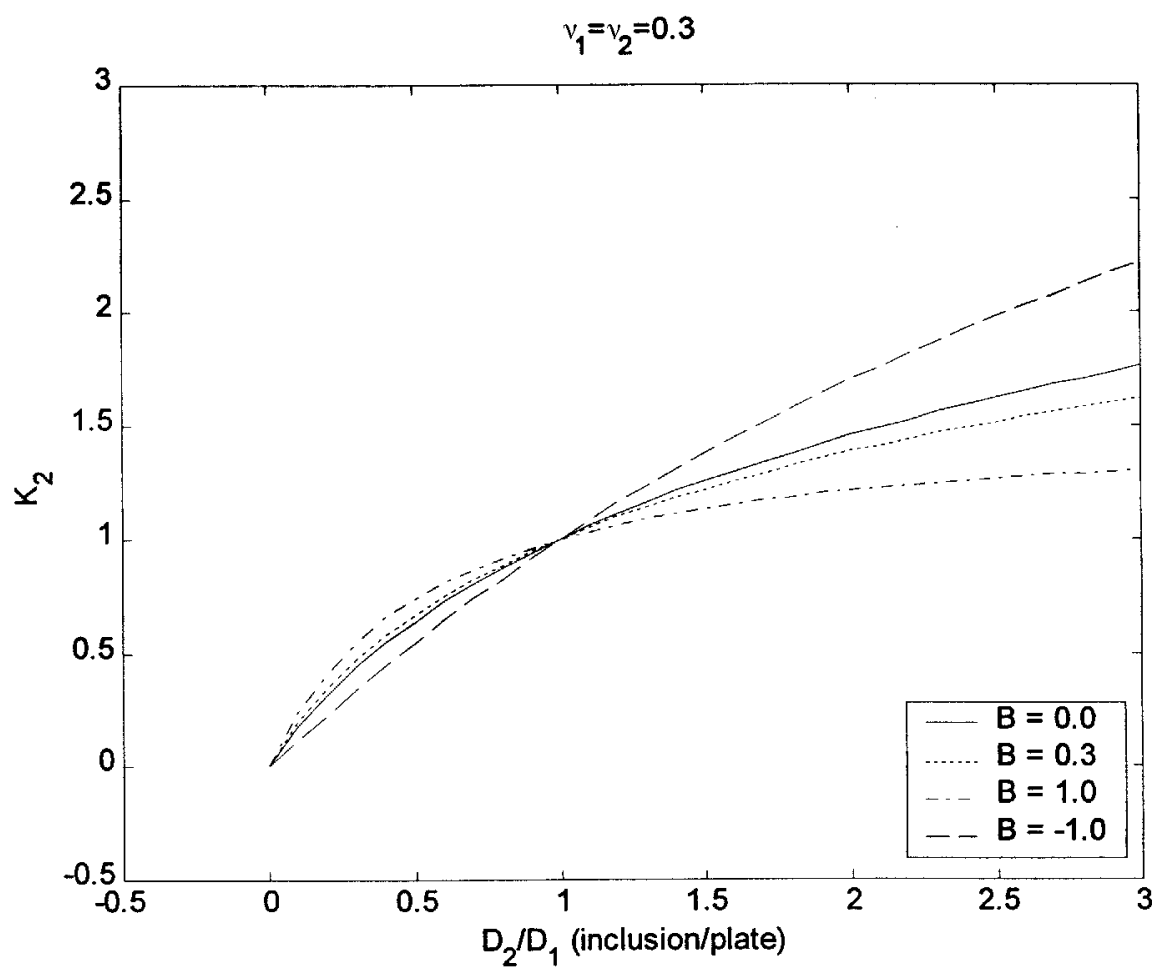

Fig. 3 Dimensionless maximum circumferential bending moment in the inclusion as a function of the ratio of the flexural rigidity of the inclusion to that of the plate

numerical example, this case is calculated: $v_{1}=0.3$, $v_{2}=0.1$ and $B=0$. The results are $K_{1}=0.9653$ and $K_{2}=1.0823$. Thus, the stress in the plate is slightly relieved while the stress in the inclusion is somewhat elevated. In contrast, if Poisson's ratios are reversed, i.e. $v_{1}=0.1, v_{2}=0.3$ and $B=0$, the resulting values are $K_{1}=1.0008$ and $K_{2}=0.9466$, i.e. $K_{1}$ is larger but $K_{2}$ is somewhat lower.

\section{REFERENCES}

1 Bickley, W. G. The effect of a hole in a bent plate. Phil. Mag., 1924, 48, 1014-1024.

2 Bert, C. W. Generalized bending of a large plate containing a circular hole. Mech. Res. Commun., 1988, 15, 55-60.

3 Goland, M. The influence of the shape and rigidity of an elastic inclusion on the transverse flexure of thin plates. J. Appl. Mechanics, 1943, 10, A69-A75. 